\title{
Mechanical damage numerical analysis of filter bags in bag type dust collector
}

\author{
Yanghou Chen ${ }^{1, a}$ \\ ${ }^{1}$ Department of mechanical and electrical engineering, School of mechanical and electrical \\ engineering, Weifang University, Weifang, China \\ achenyanghou@126.com
}

\begin{abstract}
Keywords: Filter bag; Dust collector; Mechanical damage; Finite element numerical simulation; Mesh generation
\end{abstract}

Abstract. This paper presents a new reason of the mechanical damage principle of pulse bag type dust collector bag. Three dimensional main structure of dust collector is established. And finite element numerical simulation is given. Also according to the actual situation of customer feedback, the flow field distribution in the upper and the two sides of the dust collector is uneven due to the disturbed flow at the bottom. The different flow rate caused by dust particles on the bag are not the same, which leads to lower dust and the wear of the bag is different. And some appropriate advices of dust collector are given.

\section{Introduction}

Pulse bag filter is widely used in industry because of its low running resistance, high dust removal efficiency, and small floor area and so on. In the research of dust collector, numerical simulation has become an effective research tool. At present, a part of the bag type dust collector bag breakage phenomena occur from time to time. If this is not handled in time, it is easy to cause more damage and environmental pollution. In order to study bag mechanical damage theory, numerical simulation 3D modeling of WeiFang Environmental Protection Equipment Co., Ltd. is made.

The dust collector has been improved by proper structure. There is still uneven wear bag and uneven bottom dust accumulation in the use of the process. The distribution and flow of gas in the dust collector are simulated for further analysis of the mechanical damage cause and the improvement and optimization of the internal structure.

\section{Main structure of simulated bag type dust collector}

WeiFang Environmental Protection Equipment Co., Ltd. FSMC precipitator main component has the following several parts: ladder, top of the fence, a small ladder, operation table, spray blowing and gas path system, brought out a wind pipe, central assembly, middle assembly, lower assembly and screw conveyor and so on.

In the main structure of the dust collector, the upper part, the middle part and the lower part are assembled to be responsible for the transport and distribution of the gas. The main work of the numerical simulation of fluid is aimed at this part of the structure.

\section{Finite element simulation of dust collector}

Due to the complexity of the structure, the bag filter of this company is modeled by 3D software Pro/Engineer. The model made by Pro/Engineer is repaired by using Gambit of Fluent. The division of the grid and the corresponding boundary conditions also set in the software of Fluent.

Establishment of model. The model of the lower part of the dust collector is established. The entity model established using $\mathrm{mm}$ as a unit is shown in Fig.1. External air inlet and internal split structure of dust collector also are shown in Fig.1. This kind of structure still has the problem of uneven wear and uneven distribution of dust. 


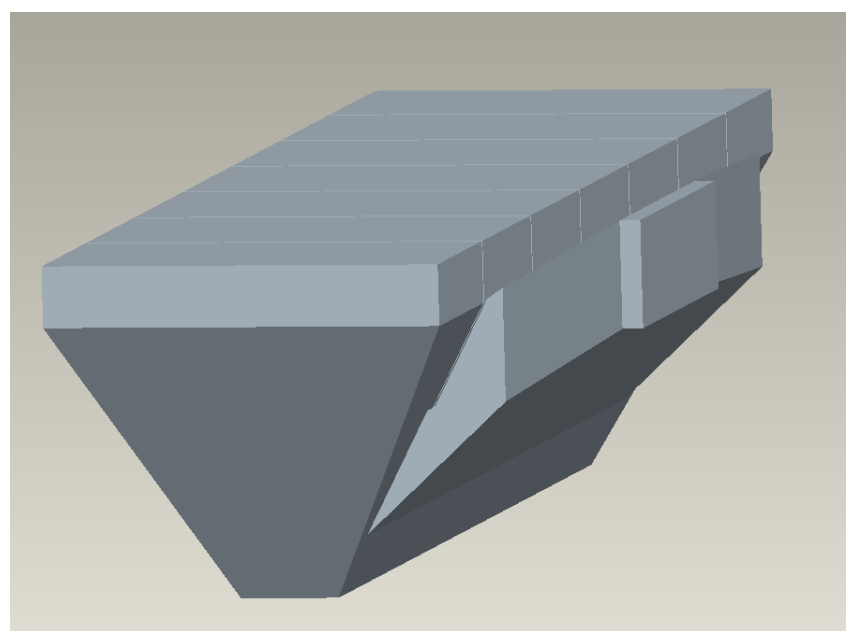

Fig.1. Solid model of dust collector

Mesh generation. Mesh is generated in the Gambit after repairing of the excess surface, line and irregular geometry. The model is properly trimmed before mesh generation. The Edge of model is divided first. Then the Surface and the Volume are meshed. Mesh generated of Volume is shown in Fig.2.

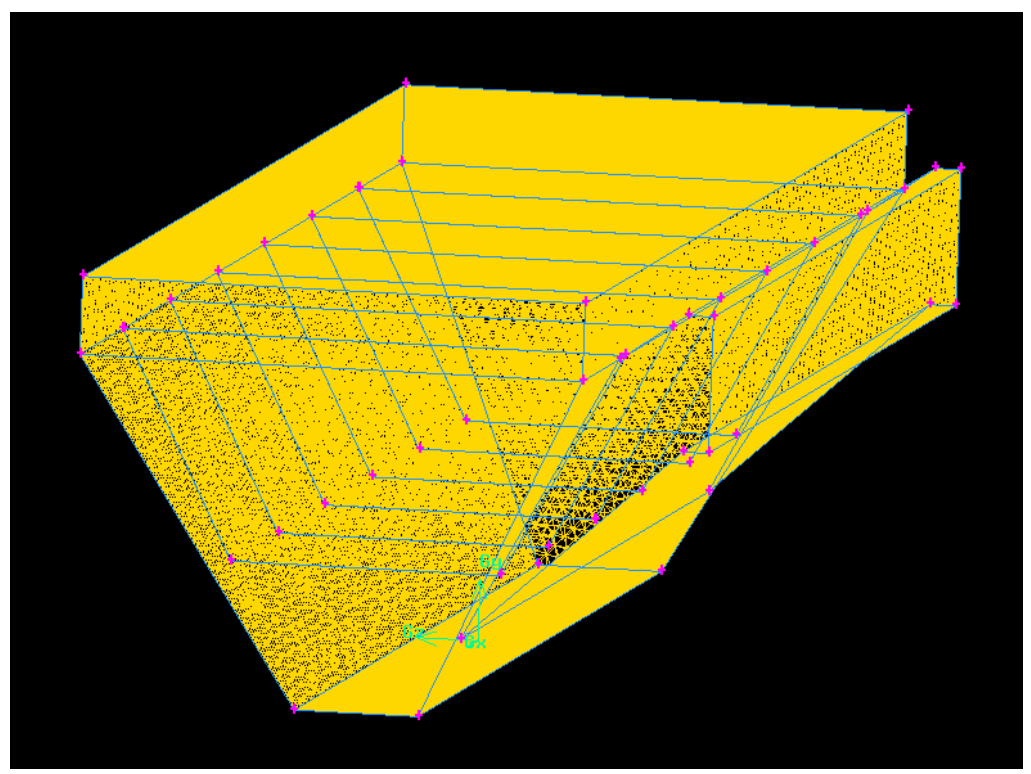

Fig.2. Volume grid of model

Fig. 2 is the mesh model, and the grid number of the whole model is 1020581 .

Boundary condition initialization. The boundary conditions are set according to the field conditions of the dust collector. The corresponding processing and grid inspection are carried out by using the software of Fluent. The easiest problem in the grid check is that the volume of the grid is negative. If the minimum volume is negative, the mesh is needed to be repaired to reduce the non physical dispersion of the solution domain. The model with inlet and outlet after grid check is shown in Fig.3.

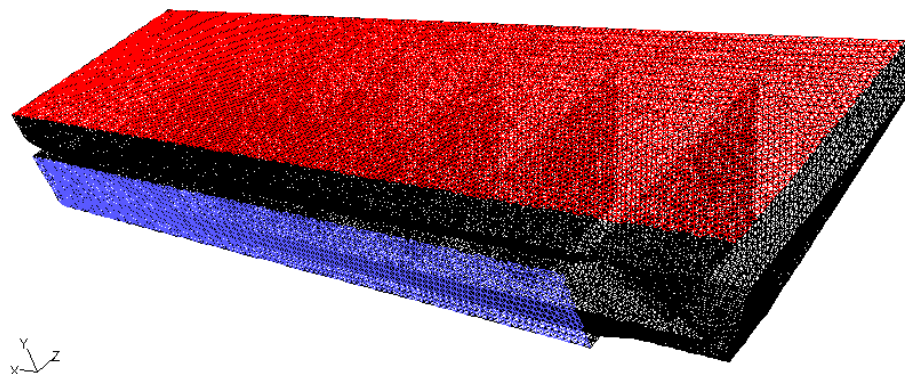

Fig.3. Model after grid check 
Fluid model selection. Fluid simulation settings are as follows: Operating Pressure:101325 Pascal; Mass Flow Specification Method: Mass Flow Rate, Mass Flow-rate $14.33 \mathrm{Kg} / \mathrm{s}$ 。Outlet vent: Gauge Pressure 101325 Pascal; Turbulence Specification Method: k and Epsilon。

\section{Results of fluid simulation}

The inlet flow and outlet flow are basically the same after 400 iterations. And the residual error tends to be stable, so the internal fluid can be considered to be in a stable state. The pathlins colored by velocity magnitude are shown in Fig.4 (a) and (b) in different viewing angles. The pathline distribution of the middle separated area is more uniform than both ends. And the velocity in this area is relatively large.

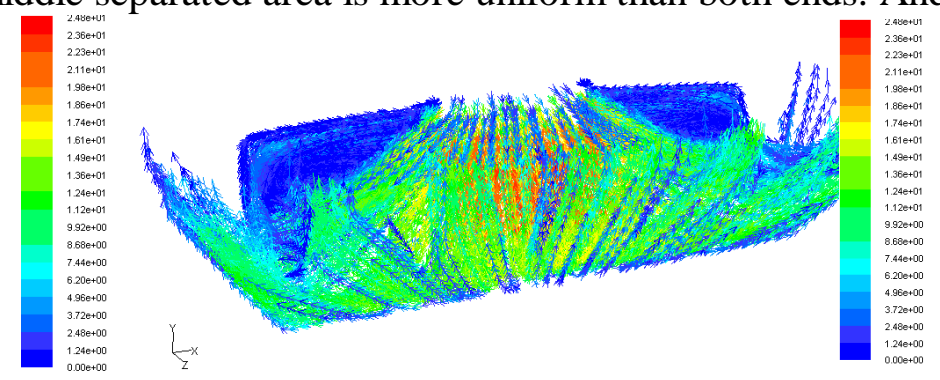

(a)

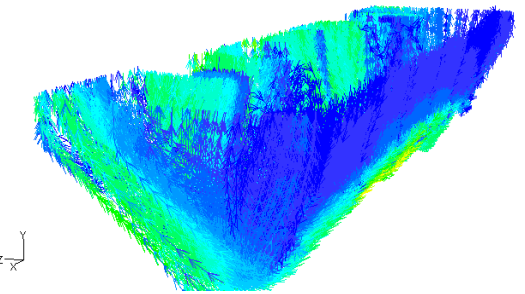

(b)

Fig.4. Pathlines colored by velocity magnitude (shown in different angles)

The velocity images of different cross sections are shown in Fig.5 (a) and (b). The symmetrical cross section also included in the figure.
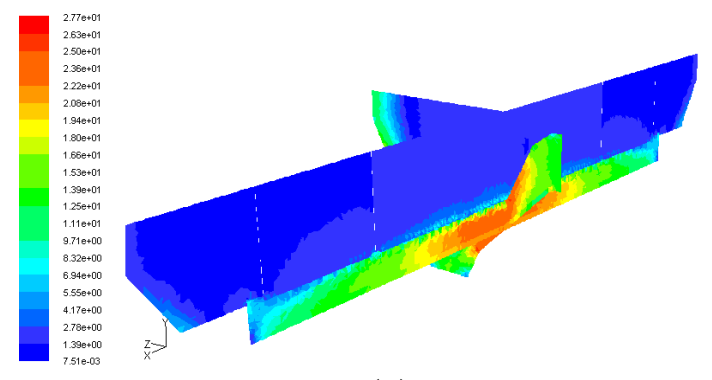

(a)

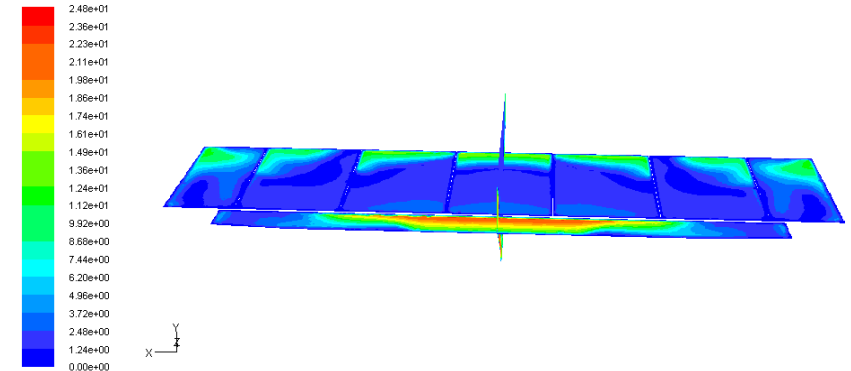

(b)

Fig.5. Multi section velocity image (filled)

In order to find out the specific velocity at different locations, $\mathrm{Y}$ axial velocity distribution in a section is shown in Fig.6.

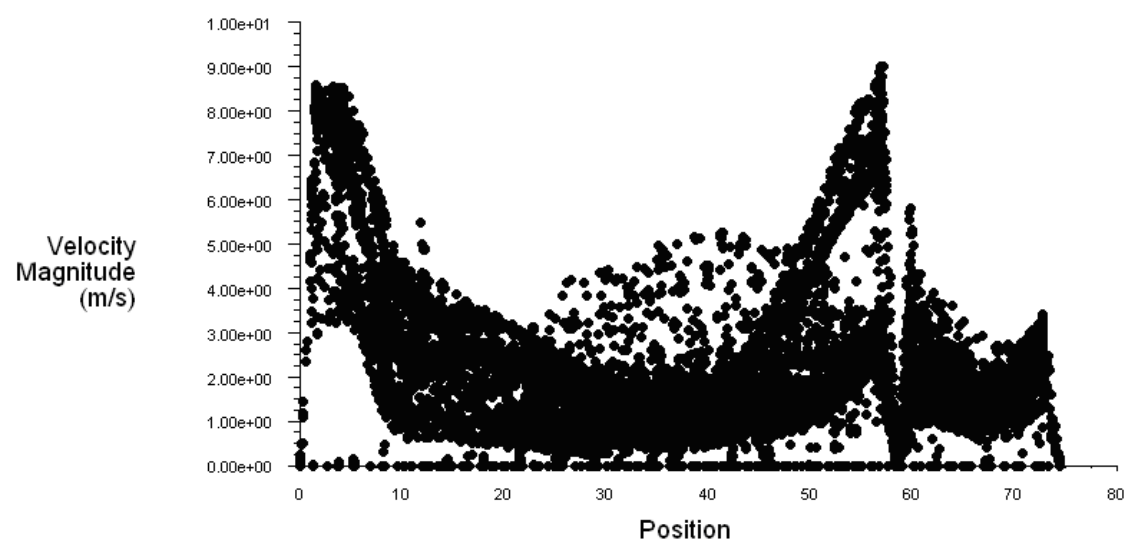

Fig.6. Velocity distribution of $\mathrm{Y}$ axial in a section

\section{Discussions}

If the working time of the dust collector and the actual working conditions of the upper bag are not considered, the air in the area is much more than the two ends because the middle part is more close to the inlet of the mixed gas. But the actual situation is that the two ends of the filter dust is more than the 
middle area, which needs further analysis of the specific circumstances of the gas flow field in the pocket area.

The dust collector has been improved by proper structure. There is still uneven wear bag and uneven bottom dust accumulation in the use of the process. The distribution and flow of gas in the dust collector are simulated for further analysis of the mechanical damage cause and the improvement and optimization of the internal structure.

From the above simulation results and show that the dust collector in the middle part of the mixed gas velocity is larger. But its velocity distribution is more uniform. On the whole, the wear of the bag should be more serious in the middle. The result is that both ends of the dust accumulation are greater than the middle part. And the damage of both ends of the bag is early than in the middle part.

\section{Conclusions}

After working for a period of time, due to the tiny and adhesion of dust and ash, the space of the bag will be blocked by dust and ash. Bag is blocked and permeability decline. And the dust and ash attached to the cloth bag will be shaken down because of the internal dynamic gas. The distribution of gas in the two ends is more uneven than in the middle of the area. Disturbed gas flap bag frequency higher, making the cloth bag attached dust more easily fall off, increase the bag permeability. And this cause the relative motion and friction between the cloth bag and the supporting frame are increased. So the wear of the bag is increased. Therefore, if we want to make the entire dust collector wears uniform, the need to improve the structure of the bag and the bag of the supporting frame.

\section{Acknowledgements}

This work was financially supported by the Shandong Natural Science Foundation (ZR2014DL010), and Project of domestic visiting scholars of young teachers of higher education institutions in Shandong Province.

\section{References}

[1] Wang Haoming, Numerical simulation of gas solid two phase flow model of lattice Boltzmann and the process of fiber trapping particles, Doctoral dissertation, (2013, In Chinese).

[2] Bin Wang, Liqin Liu and Yougang Tang, CFD Simulation of the Vertical Motion Characteristics of the Moonpool Fluid for the Truss Spar, J. Marine Sci. Appl. 13: 92-98. (2014).

[3] Tang, G.H., Lu, Y.B.: A resistance model for Newtonian and power-law non-Newtonian fluid transport in porous media. Transp. Porous Media 104, 435-449 (2014).

[4] Di Federico, V., Pinelli, M., Ugarelli, R.: Estimates of effective permeability for non-Newtonian fluid flow in randomly heterogeneous porous media. Stoch. Environ. Res. Risk Assess. 24(7), 1067-1076 (2010).

[5] Information on http://www.peraglobal.com/index.html

[6] Chen yanghou, Chen Guoming, Lin Hong, Scour prevention of offshore platform pile foundation and CFD simulation, Journal of China University of Petroleum (Edition of Natural Science), Vol. 33. 112-116 (2009, In Chinese)

[7] Information on http://www.meilele.com/bbs/thread-31639-1-1.html

[8] Kuznetsov, Mikhailik, ksieko ,Rusanov, Modeling of the hydrodynamics of a cyclonic rotational dust collector of increased efficiency. Journal of Engineering Physics \& Thermophysics. Mar2012, Vol. 85 Issue 2, p349-355. 